\title{
What impact would an HIVIAIDS vaccine have on the HIVIAIDS epidemic in Kenya?
}

\author{
Omu Anzala ${ }^{1^{*}}$, Gaudensia Nzembi Mutua ${ }^{1}$, Fredrick Jack Odia Oyugi ${ }^{1}$, \\ Bashir Farah Mohamed ${ }^{1}$, Thomas Achia ${ }^{2}$, John Stover ${ }^{3}$ \\ ${ }^{1}$ Kenya AIDS Vaccine Initiative, Department of Medical Microbiology, University of Nairobi, Nairobi, Kenya; \\ *Corresponding Author: oanzala@kaviuon.org \\ ${ }^{2}$ School of Mathematics, University of Nairobi, Nairobi, Kenya \\ ${ }^{3}$ Futures Institute Glastonbury, 41A New London Turnpike, Glastonbury, USA
}

Received 28 July 2012; revised 17 August 2012; accepted 25 September 2012

\begin{abstract}
Objective: To estimate the potential impact of an HIVIAIDS Vaccine in Kenya. Design: The Kenyan HIVIAIDS epidemic was modeled using the most current data from national sources including epidemiology and behavioral surveillance. The model's baseline projection was validated against adult HIV prevalence at antenatal clinics and general population surveys. The model was used to analyze the effects of scaling up current prevention programs and adding potential HIV vaccines with varying levels of effectiveness and coverage. Results: Even with full scale-up of currently available prevention, care and treatment programs, new infections will continue to burden Kenya. The introduction of a partially effective AIDS vaccine could significantly alter the trajectory of the epidemic. Conclusion: The game changing impact that an AIDS vaccine could have on the AIDS epidemic in Kenya underscores the importance of sustaining political support and financial investment to accelerate HIVIAIDS vaccine research and development.
\end{abstract}

Keywords: HIV; Vaccine; Modelling

\section{INTRODUCTION}

A national household survey conducted in Kenya in 2007 [1] found that $7.8 \%$ of Kenyans between the ages of 15 and 49 are infected with HIV. The incidence of new infections probably peaked around the year 2000 and has declined somewhat since then. Current prevention efforts including behavior change communication (BCC), voluntary counseling and testing (VCT), prevention of motherto-child transmission (PMTCT), use of condoms, abstinence, circumcision, and home-based HIV testing have contributed to lowering the number of new infections.
While progress has been made in reducing new infections in urban areas, incidence remains high in rural populations and among most-at-risk populations. With about $120,000(71,000$ - 155,000) new infections each year [2], there is a critical need for new prevention technologies, in particular an HIV vaccine, to combat and ultimately end the epidemic in Kenya.

As of September 2010, there were 23 ongoing HIV vaccine clinical trials [3]. The question on the minds of researchers, funding organizations, policymakers and other stakeholders is whether any of the candidate vaccines currently in development will induce full protection or lower viral load, delay disease progression and reduce the risk of HIV transmission in those individuals who are vaccinated and become infected with HIV. Although, there is currently not an AIDS vaccine available for use today, it is important to study the potential impact now in order to understand the contribution a vaccine could make in the fight against HIV/AIDS and the efforts required to support vaccine development.

This study investigated the potential impact of HIV vaccines with varying levels of effectiveness and coverage in preventing new infections in Kenya in order to provide policymakers and other stakeholders with a better understanding of how HIV vaccines could be used as part of a comprehensive strategy to end the epidemic; to prepare and adopt appropriate policies to support vaccine development and eventual introduction.

\section{BACKGROUND}

Mathematical modeling and computer simulation of the impact of vaccination and other infectious disease control efforts have become powerful tools for health policy evaluation, policy dialogue, and advocacy. A number of such mathematical and computer simulation models have been developed specifically to estimate the impact of a vaccine on the AIDS epidemic [3].

The International AIDS Vaccine Initiative (IAVI) and 
the Futures Institute have worked together to develop a mathematical model to study the potential impacts of an HIV vaccine on the pandemic both globally [4], and in countries such as Kenya, China, Uganda and Brazil [5]. In Kenya, IAVI developed this research in collaboration with the Kenya HIV and AIDS Research Coordinating Mechanism (KARSCOM) and a team of researchers at the Kenya AIDS Vaccine Initiative (KAVI) led the technical work with assistance from the Futures Institute.

\section{METHOD}

\subsection{The Model}

Estimates of the potential impacts of HIV vaccines were made using the HIV vaccine model within the Spectrum Policy Modeling System [6]. This model uses readily available data to reproduce key dynamics of the HIV epidemic including the most current data from national sources and epidemiological and behavioral surveillance data. Demographic information used in modeling was obtained from DemProj, another module within Spectrum, based on assumptions about base year, population size, fertility and mortality from the United Nations Population Division. Various published sources provided additional demographic, health and behavioral data [7-10]. The model's baseline projection was validated against the adult HIV prevalence as recorded in HIV surveillance at antenatal clinics in two general population surveys [11,12].

The model simulates the adult population ages 15 - 49 . The population is divided by sex, but is not further stratified by age. Individuals were classified into distinct compartments (states) based on their behavior and HIV and vaccination status.

People were categorized into one of five risk groups as follows:

- Low-risk heterosexual (LRH): those with one steady sexual partner.

- Medium-risk heterosexual (MRH): those with multiple partners.

- High-risk heterosexual (HRH): commercial sex workers and their clients.

- High-risk: Men-who-have-sex-with-men (MSM).

- High-risk: Injecting drug users (IDUs).

Individuals enter the model at age 15 as not sexually active (NSA) and remain in that category until the median age at first sex, at which time they are moved into one of the risk groups. An individual in the medium-risk, high-risk or injecting drug user risk group may remain in that group throughout the projection or can move to a lower-risk category. This movement is particularly important for female sex-workers since they may be sexworkers for only a relatively short period that is 5 to 10 years of their adult life.
The behavioral parameters employed in the model include: sexual acts per partner, age at first sex, number of sexual partners, condom use, force of infection for IDUs and proportion married. The behavioral parameters were obtained from a secondary data search $[13,14]$ and extrapolated where information was not available.

Once infected, an individual then enters the primary infection stage for a period of six months. People in the primary infection stage are more infectious than those in other stages. The individual then enters the asymptomatic stage and remains there for ten years. The asymptomatic stage is characterized by a low level of infectiousness and is followed by the final symptomatic stage where infected individuals remain for two years before dying. Both the infected and uninfected individuals may die from other causes.

\subsection{Vaccine Characteristics}

Although the level of protection that first-generations vaccines could provide is still unknown, scientists believe they might be only partially effective in preventing HIV infection. Based on the vaccine candidates currently being tested, a vaccine could employ a combination of three mechanisms to fight the virus: 1) reduce susceptibility to infection (via protective immunity); 2) reduce infectiousness of vaccinated individuals; and 3) slow progression to AIDS morbidity and death (modifying disease).

Reduce susceptibility: A vaccine administered to HIVnegative people could provide protection by reducing their susceptibility to a persistent infection, effectively clearing the body of HIV and thus preventing disease; this is the commonly understood action of a vaccine.

If the vaccine action is set to "take" in the model, then a certain portion of those vaccinated are fully protected from acquiring HIV - that is, the vaccine is completely effective for some people and has no effect on others. The percentage of people protected is determined by the vaccine's efficacy, while the portion not protected is fully exposed to the risk of infection.

In a "degree" type of vaccine, all of those who are vaccinated are exposed to a risk of infection that is reduced by the efficacy of the vaccine; this causes a reduction in susceptibility for everyone who is vaccinated.

Reduce infectiousness: A preventive vaccine could also keep the amount of virus in a person at a low level so that he or she is less likely to infect others. The model calculates the reduction in the average probability of transmission resulting from this type of efficacy and coverage of the vaccine and applies this to all contacts with susceptible populations.

Modifying disease: A vaccine given to an HIV-negative person could also provide a benefit by greatly slowing progression to AIDS morbidity. The model implements this by lengthening the asymptomatic period for 
those vaccinated who obtain this benefit but does not change the length of the primary or symptomatic stages.

In all cases, it is assumed that the vaccine is effective only when the recipient is HIV-negative. Although a therapeutic effect is theoretically possible, the model does not attempt to capture any kind of therapeutic actionthat is, there is no assumed benefit to people who are HIV-positive when vaccinated. The duration of vaccine protection could range from very short (a few years) to lifetime.

\subsection{Vaccine Scenarios}

Through consultations with leading researchers and policymakers in Kenya, three plausible scenarios were constructed to reflect current understanding of vaccine science and the Kenyan response to the epidemic. The three scenarios looked at the impact of a vaccine with varying levels of effectiveness (30\%, 50\% and 70\%) and population coverage $(30 \%, 50 \%$, and $70 \%$ of the adult population 15 - 49 years of age). In the model, the vaccine is assumed to be introduced in the year 2020 and coverage scales up from 0\% in 2019 to the maximum coverage by the year 2025. Coverage then remains constant until 2050. All scenarios assume a vaccine with "take" action and protect and individual for a duration of 20 years.

In the three main scenarios, vaccination coverage varies by target population group but the vaccine efficacy is the same across all population groups. The model assumed that the vaccinations would be administered without HIV testing.

\subsection{Targeted Vaccination Strategies}

When a vaccine first becomes available, a key policy question will be whether to move forward with a vaccination strategy that covers the general population or, instead target specific population groups with increased vulnerability to HIV such as men who have sex with men (MSM), injecting drug users (IDUs), heterosexuals with multiple partners and sex workers and their customers. The study explored the impact of four vaccination strategies. All strategies use the Medium (50\%) efficacy vaccine (described in Table 1).

\subsection{Limitations of the Model}

The model is intended to replicate the major dynamics of the epidemic in Kenya that are relevant to the potential impact of an HIV vaccine. A number of simplifications were made in order to work with available data. The major limitations were:

- The adult population is not age structured; the model considers only the adult population 15 - 49 as a single age group;
- The model only allows people that start in medium or high-risk groups to switch to lower-risk groups. It does not allow switching in and out of high-risk groups over a person's lifetime;

- The model makes deterministic projections and thus does not represent the uncertainty associated with projections;

- Mathematical models are not designed to replace information obtained from surveillance. However they provide a framework to analyze and communicate results.

\section{RESULTS}

A baseline projection was prepared for Kenya assumeing that no vaccine becomes available, but coverage for other prevention interventions and treatment increased significantly. Table 2 shows the projections of new in fections, incidence, prevalence of HIV/AIDS and adult population under the assumption that current preventive measures are improved upon from 1990 to 2050.

\subsection{HIVIAIDS Vaccine Scenarios}

The base projection (Figure 1) shows the adult HIV incidence from 1980-2050 without the introduction of a vaccine (incidence is the percentage of the uninfected population that becomes infected each year). Without a vaccine, adult incidence is expected to decline rapidly

Table 1. Potential vaccination strategies with medium (50\%) efficacy vaccine.

\begin{tabular}{cc}
\hline \multicolumn{2}{c}{ Potential vaccination strategies with medium (50\%) } \\
efficacy vaccine
\end{tabular}

Table 2. Baseline projections.

\begin{tabular}{ccccc}
\hline Year & $\begin{array}{c}\text { New } \\
\text { infections }\end{array}$ & Incidence & Prevalence & $\begin{array}{c}\text { Number of } \\
\text { adults }\end{array}$ \\
\hline 1990 & 59,339 & 0.62 & 2.67 & $9,873,624$ \\
2000 & 128,590 & 0.92 & 5.76 & $14,827,138$ \\
2010 & 147,560 & 0.79 & 7.10 & $20,126,852$ \\
2020 & 127,419 & 0.52 & 6.69 & $26,439,312$ \\
2030 & 146,324 & 0.46 & 5.73 & $34,231,152$ \\
2040 & 159,181 & 0.41 & 5.16 & $41,300,200$ \\
2050 & 170,406 & 0.37 & 4.74 & $48,068,916$ \\
\hline
\end{tabular}




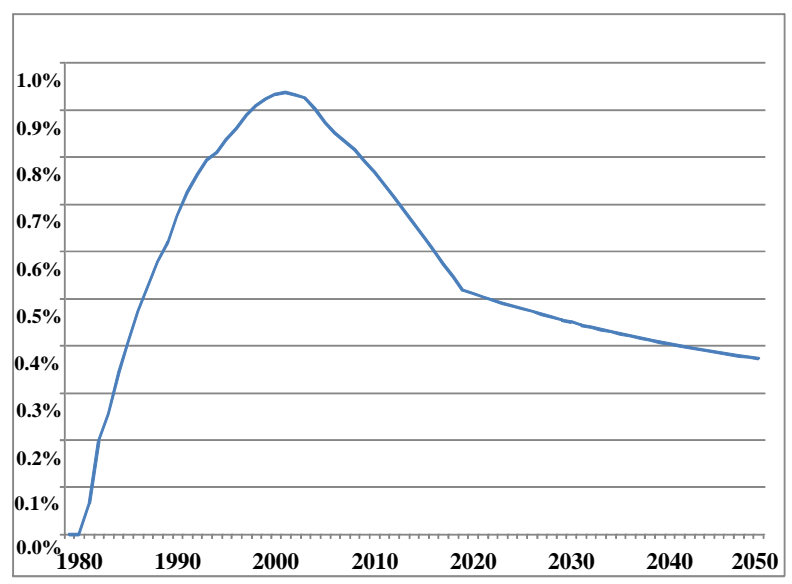

Figure 1. Adult HIV incidence (Base projection).

until the year 2020 as a result of full scale up of treatment and prevention inventions. Maximum coverage of treatment and prevention interventions is reached in 2020 and incidence declines more slowly after that. Even though incidence declines after 2020 the number of new infections actually increases because of population growth.

The scenarios outlined in Figure 2 assume that a vaccine is introduced in the year 2020 and maximum coverage is reached by 2025 because of the time need to scale up coverage. The level of coverage is assumed to increase as vaccine efficacy increases. A higher efficacy vaccine is likely to be implemented more broadly by governments and is likely to have greater uptake among individuals. All scenarios assume the continuation and scale-up of existing treatment and prevention intervenetions by 2020, consistent with the goals set out in Kenya's National AIDS Plan.

The baseline scenario shown in blue (Figure 2) projects the future trajectory of the epidemic in Kenya if a vaccine is not introduced and existing treatment and preventions interventions are scale-up significantly.

Without a vaccine, and even with major scale up of existing treatment and prevention intervention to maximum coverage, the number of new infections is projected to increase after 2020 due to population growth.

Results generated by this analysis show that an AIDSvaccine could substantially alter the course of the epi-

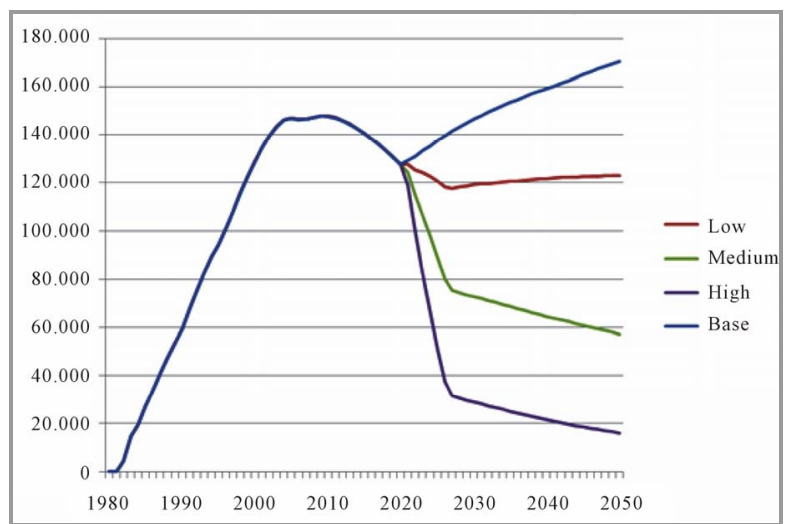

Figure 2. New Adult HIV Infections in Kenya, By Year and Vaccine Scenario, 2020-2050.

demic in Kenya and reduce the number of new infections, even if vaccine efficacy levels are relatively low.

A medium efficacy vaccine scenario (one that reduces that chance of becoming infected by $50 \%$ ) given to half of the adult population is forecast to have a substantial impact by preventing around 65\% of new HIV infections and averting 850,000 deaths from 2020 to 2050. A vaccine with higher efficacy (70\%) given to more than half of the population (70\%) would have an even greater impact on the epidemic, averting an estimated $90 \%$ of new infections and 1.2 million deaths from 2020 to 2050. Table 3 shows the vaccine efficacy.

\subsection{Targeted Vaccination Strategies}

Once an effective HIV vaccine becomes available it would probably be made available to all those who want it, but, especially in the early stages of vaccine roll-out, there may be limitations in vaccine supply and resources that make it difficult to reach everyone at once. Since everyone is not at equal risk of infection, four targeted vaccination strategies were explored. Table 4 shows the number of infections averted from 2020 to 2050 and the number of vaccinations per infection averted in the Medium vaccine scenario and the following targeting scenarios:

- MEDIUM: Vaccinating $50 \%$ of all adult population groups (15 to 49 years old);

- HIGHER COVERAGE 80\%: Vaccinating 80\% of

Table 3. Vaccine efficacy.

\begin{tabular}{ccccc}
\hline Vaccine scenarios & Efficacy & $\begin{array}{c}\text { Percentage of } \\
\text { population given vaccine }\end{array}$ & $\begin{array}{c}\text { New infections } \\
\text { averted, 2020-2050 }\end{array}$ & $\begin{array}{c}\text { Deaths averted, } \\
\mathbf{2 0 2 0 - 2 0 5 0} \\
\text { reduction in new infections }\end{array}$ \\
\hline LOW & $30 \%$ & $30 \%$ & 1.4 million & 409.000 \\
MEDIUM & $50 \%$ & $50 \%$ & 2.3 million & 850.000 \\
HIGH & $70 \%$ & $70 \%$ & 3.5 million & 1.233 .000 \\
\hline
\end{tabular}


Table 4. Potential impact of vaccination strategies in Kenya.

\begin{tabular}{|c|c|c|c|c|}
\hline Vaccine Strategy & $\begin{array}{l}\text { Percentage Reduction in } \\
\text { New Infections, 2020-2050 }\end{array}$ & $\begin{array}{c}\text { New Infections } \\
\text { Averted, 2020-2050 }\end{array}$ & $\begin{array}{l}\text { Vaccinations } \\
\text { Required, 2020-2050 }\end{array}$ & $\begin{array}{l}\text { Vaccinations Per } \\
\text { Infections Averted }\end{array}$ \\
\hline $\begin{array}{l}\text { HIGHER COVERAGE } \mathbf{8 0} \% \\
\text { 80\% coverage of medium-and high-risk } \\
\text { and } 50 \% \text { of low risk groups }\end{array}$ & $72 \%$ & 2.67 million & 69.4 million & 26 \\
\hline $\begin{array}{l}\text { MEDIUM-AND HIGH-RISK ONLY } \\
50 \% \text { coverage of medium-and high-risk } \\
\text { groups }\end{array}$ & $26 \%$ & 950,000 & 8.6 million & 9 \\
\hline $\begin{array}{c}\text { HIGH-RISK ONLY } \\
50 \% \text { coverage of high-risk groups }\end{array}$ & $3 \%$ & 120,000 & 1.4 million & 12 \\
\hline $\begin{array}{c}\text { MEDIUM } \\
50 \% \text { coverage of adult population }\end{array}$ & $65 \%$ & 2.4 million & 62.4 million & 26 \\
\hline
\end{tabular}

individuals with behaviors placing them at high and medium risk of infection; and vaccinating $50 \%$ of the population with low risk of contracting HIV;

- MEDIUM- AND HIGH-RISK ONLY: Vaccinating $50 \%$ in the medium- and high-risk populations and no coverage of low-risk groups;

- HIGH-RISK ONLY: Vaccinating 50\% of only highrisk individuals, with no vaccination coverage of medium- or low-risk groups

According to the model, the higher coverage scenario, which aims to cover $80 \%$ of medium and high risk populations along with $50 \%$ of low risk groups, would be the most effective vaccination strategy, averting nearly 2.7 million new infections from 2020-2050. However, it may be challenging to reach $80 \%$ of the medium- and high-risk population with the vaccine and this strategy requires the largest number of vaccinations. In resourcelimited setting, the most cost effective strategy, in terms of number of vaccine per infection averted would be a medium- and high-risk only strategy that aims to cover $50 \%$ of medium- and high-risk groups. This strategy would require only one-eighth of the number of vaccinetions as the higher-coverage strategy and result in a $26 \%$ reduction in new infections from 2020-2050.

The data show that the lowest impact on curbing the epidemic resulted when targeting only high-risk groups for vaccination, which would avert only 3\% of new infections. Because Kenya's epidemic is generalized and affects all segments of the population, it is critical that a vaccination strategy covers a wide cross section of the population, rather than solely targeting those at highest risk of infection, in order to have a significant impact on the national epidemic (Figure 3).

\subsection{Cost Analysis}

Although it is unclear at this time what the full cost of a vaccine will be, preliminary cost analyses suggest that

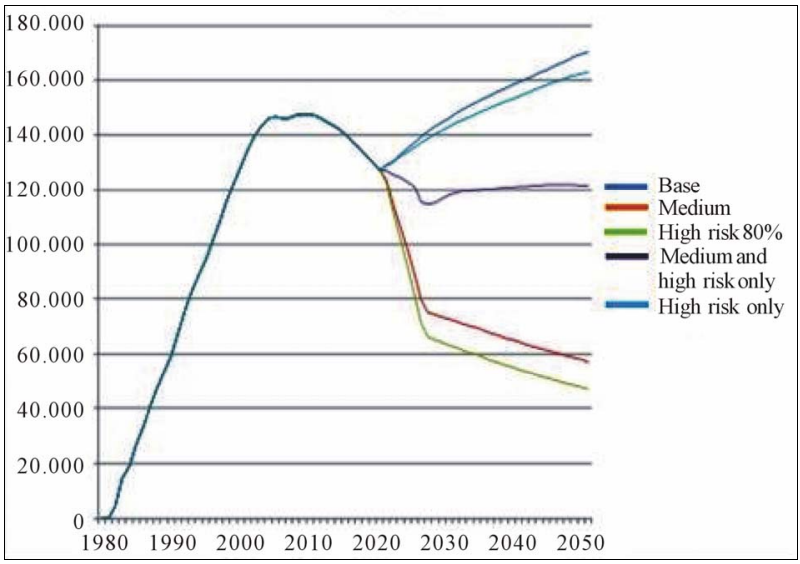

Figure 3. Number of new HIV infections with different targeting strategies.

AIDS vaccines would result in significant savings when compared to growing costs of care and treatment. The maximum price at which a vaccine could be considered cost-saving can be calculated using several different approaches. In our analyses we examined the cost of a vaccine compared to the present value of future treatment costs, as an infection averted will mean that a person will not need ART in the future. In Kenya, the lifetime costs of maintaining a person on care and treatment who becomes infected today would be about $\$ 7000$ in present value. Therefore, any intervention that prevents HIV infection for less that $\$ 7000$ would not only be cost-effective, but cost-saving.

In the medium efficacy vaccine scenario, impact modelling estimates that approximately 62.4 million vaccinations would be required to avert 2.4 million infections from 2020 to 2050. This means that approximately 26 vaccinations are needed to avert one infection, In comparison to ART, a medium-efficacy vaccine could cost up to $\$ 269$ per vaccination (US\$7000 divided by 26) and still result in cost savings when compared to the care and treatment costs that are avoided. 


\section{DISCUSSION}

The HIV epidemic continues as a major health challenge in Kenya more than 85,000 [15] deaths per year in spite of efforts to expand the coverage of prevention programs. Since 2003, the proportion of HIV infected individual accessing ART has been expanding. In 2009 $70 \%$ of those most in need were receiving ART [16]. Over the past 10 to 15 years a number of research organizations have looked at the potential impact of a vaccine on the AIDS epidemic [6]. And, even though the development of an effective HIV/AIDS vaccine is still a number of years away, the kind of impact an HIV/AIDS vaccine is likely to have remains an important question to funding organizations and policy makers.

Scaling-up all currently available treatment and prevention options will make an impact on the epidemic in Kenya, but in Kenya going by a model we will still experience over 170,000 new infections per year by 2050 . Thus there is an urgent need to develop a vaccine.

In the medium efficacy vaccine scenario, even a vaccine with efficacy of $50 \%$, and with $50 \%$ population coverage would have a significant impact in reducing the number of new infections, HIV prevalence, incidence and HIV/AIDS-related mortality by the year 2050. Under this kind of a scenario, approximately 850,000 cumulative HIV-related deaths will averted over a 30 -year period.

Apart from the effectiveness of an HIV vaccine, other issues that are important to stakeholders and policy makers are the infrastructure required to achieve the expected results predicted by the model and costs involved in the manufacture of the vaccine. The costs include the production of millions of vaccine doses, cost of vaccination coverage and the logistics involved. If the vaccine targets HIV negative individuals, additional costs for testing large numbers of people will have to be taken into consideration.

Sex workers, men-who-have-sex-with-men, and intravenous drug users are high transmitters of HIV $[2,17]$. Men and women with multiple sexual partners (the medium-risk population in the model) have lower individual risk than those in the highest-risk population but there are many more people in the medium-risk category, making it very important to the control of the epidemic. It is likely that these groups will continue to be important to the HIV epidemic in the general population. Targeting the high- and medium-risk groups will likely be more cost effective than targeting the general population but will provide less overall impact.

The HIV/AIDS epidemic has been persistent despite massive investments in prevention programs. Behaviour change has been slow and unsustainable on an individual basis $[18,19]$. It is possible that the introduction of a partially effective AIDS vaccine may result in some degree of behavioral disinhibition, which occurs when vaccinated individuals believe they are protected from HIV infection and therefore increase behaviors that put them at greater risk of exposure to HIV. To mitigate against behavioral disinhibition, broad-based education and awareness campaigns with tailored messaging will need to be properly crafted and delivered prior to the introduction of a vaccine.

Findings from this research highlight the relevance of AIDS vaccines in Kenya and the importance of sustaining investments and strong policy efforts to accelerate AIDS vaccine research and development both in Kenya and internationally.

\section{ACKNOWLEDGEMENTS}

This project was sponsored fully by funds from International AIDS Vaccine Initiative (IAVI), New York, USA.

\section{REFERENCES}

[1] KAIS (2007) C Kenya AIDS Indicator Survey report. National AIDS Control Program, Nairobi.

[2] National AIDS and STD Control Programme (2010) National HIV indicators for Kenya: 2009. NASCOP/NACC, Nairobi.

[3] IAVI (2005) Modeling the impact of AIDS vaccines. International AIDS Vaccine Initiative, New York.

[4] Stover, J., Bollinger, L., Hecht, R. and Williams, C. (2007) The impact of an AIDS vaccine in developing countries: A new model and initial results health affairs. Health Affairs, 26, 1147-1158.

[5] Fonseca, M.G.P., Forsythe, S., Menezes, A., et al. (2010) Modeling HIV vaccines in Brazil: Assessing the impact of a future HIV vaccine on reducing new infections, mortality and number of people receiving ARV. PLoS One, 5, e11736. doi:10.1371/journal.pone.0011736

[6] Stover, J., Johnson, P., Zaba, B., et al. (2008) The spectrum projection package: Improvements in estimating mortality, ART needs, PMTCT impact and uncertainty bounds. Sexually Transmitted Infections, 84, i24-i30. doi:10.1136/sti.2008.029868

[7] Akwara, P.A., Madise, N.J. and Hinde, A. (2003). Perception of risk of HIV/AIDS and sexual behaviour in Kenya. Journal of Biosocial Science, 35, 385-411 doi:10.1017/S0021932003003857

[8] Angala, P., Parkinson, A., Kilonzo, N., Natecho, A. and Taegtmeyer, M. (2006) Men who have sex with men (MSM) as presented in VCT data in Kenya. XVI International AIDS Conference, Toronto, 13-18 August 2006.

[9] Auvert, B., Taljaard, D., Lagarde, E., et al. (2005) Randomized, controlled intervention trial of male circumcision for reduction of HIV infection risk: The ANRS 1265 trial. PLoS Medicine, 2, e298. doi:10.1371/journal.pmed.0020298

[10] Bailey, R.C., Moses, S., Parker, C.B., et al. (2007) Male circumcision for HIV prevention in young men in Ki- 
sumu, Kenya: A randomized controlled trial. Lancet, 369, 643-656. doi:10.1016/S0140-6736(07)60312-2

[11] NASCOP (2009) HIV prevention response and modes of transmission analysis Kenya. NASCOP, Nairobi.

[12] KDHS (2003) 2003 preliminary report. Kenya Demographic and Health Survey.

[13] Zaba, B., Pisani, E., Slaymaker, E. and Boerma, J.T. (2004) Age at first sex: Understanding recent trends in African demographic surveys. Sexually Transmitted Infections, 80, ii28-ii35. doi:10.1136/sti.2004.012674

[14] Ngugi, E.N., Plummer, F.A., Simonsen, J.N., et al. (1988) Prevention of transmission of HIV in African and the effectiveness of condom promotion and health education among prostitutes. Lancet, 2, 887-890. doi:10.1016/S0140-6736(88)92480-4
[15] UNAIDS/WHO (2008) Kenya Epidemiological Fact Sheet on HIV and AIDS, 2008. UNAIDS, Nairobi.

[16] UNGASS (2010) County report for Kenya National AIDS Control Council, Nairobi.

[17] Powers, K.A., Poole, C., Pettifor, A.E. and Cohen, M.S. (2008) Rethinking the heterosexual infectivity of HIV-1: A systematic review and meta-analysis. The Lancet, 8, 70156-70157.

[18] Know your epidemic report (2009) No. of Adults and Children receiving antiretroviral therapy (ART), 20002008.

[19] KDHS (1998) Kenya Demographic and Health Survey 1998 report, KDHS, Nairobi. 